\title{
ON THE SHORT TIME SCALE EVOLUTIONARY HISTORY OF THE CONTACT BINARY VW CEPHEI
}

\author{
I. PUSTYLNIK \\ Tartu Observatory, EE2444, Estonia
}

We study the short-time evolutionary history of the well-known contact binary VW Cep. Our analysis is based partly on the numerous $U B V$ light curves obtained at Tartu Observatory, IUE spectra, and samples from the published data. Special attention is given to the effects of asymmetry of the light curves. A higher degree of asymmetry outside the eclipses along with the significant displacements of the brightness maxima in respect to the elongation phase is interpreted as evidence that a considerable portion of the flaring source is concentrated close to the neck connecting the components. We discuss the nature of asymmetry in terms of possible mass exchange and the flare activity and compare the results of our model computations with the record of orbital period variations over the last 60 years. 